\title{
Color-Luminosity Relations for the Resolved Hot Stellar Populations in the Centers of M31 and M32
}

Thomas M. Brown

Laboratory for Astronomy \& Solar Physics, Code 681, NASA/GSFC, Greenbelt, MD, USA 20771

Henry C. Ferguson

Space Telescope Science Institute, 3700 San Martin Drive, Baltimore, $M D$, USA 21218

\section{S.A. Stanford}

Institute of Geophysics and Planetary Physics, Lawrence Livermore National Laboratory, Livermore, CA, USA 94550

Jean-Michel Deharveng

Laboratoire d'Astronomie Spatiale du CNRS, Traverse du Siphon, BP 8, F-13376 Marseille Cedex 12, France

\begin{abstract}
.
We present Faint Object Camera (FOC) ultraviolet images of the central $14 \times 14^{\prime \prime}$ of Messier 31 and Messier 32. The hot stellar population detected in the composite UV spectra of these galaxies is partially resolved into stars, and we measure their colors and apparent magnitudes. We detect 433 stars in M31 and 138 stars in M32, down to limits of $m_{F 275 W}=25.5 \mathrm{mag}$ and $m_{F 175 W}=24.5 \mathrm{mag}$. We investigate the luminosity functions of the sources, their spatial distribution, their colormagnitude diagrams, and their total integrated far-UV flux. Although M32 has a weaker UV upturn than M31, the luminosity functions and color-magnitude diagrams of M31 and M32 are surprisingly similar, and are inconsistent with a majority contribution from any of the following: post-AGB stars more massive than $0.56 M_{\odot}$, main sequence stars, or blue stragglers. The luminosity functions and color-magnitude diagrams are consistent with a dominant population of stars evolving from the extreme horizontal branch (EHB) along tracks of mass $0.47-0.53 M_{\odot}$. These stars are well below the detection limits of our images while on the zero-age EHB, but become detectable while in the more luminous (but shorter) post-HB phases. Our observations require that only a very small fraction of the main sequence population (2\% in M31 and $0.5 \%$ in M32) in these two galaxies evolve though the EHB and post-EHB phases, with the remainder rapidly evolving through bright post-AGB evolution with few resolved stars expected in the small field of view covered by the FOC.
\end{abstract}




\section{Introduction}

The spectra of elliptical galaxies and spiral galaxy bulges exhibit a strong upturn shortward of $2700 \AA$, dubbed the "UV upturn." Early explanations for the source of the UV upturn covered a wide range of candidates, including massive young stars, extreme horizontal branch (EHB) stars, planetary nebula nuclei, and several binary scenarios (see Greggio \& Renzini 1990 for a complete review). The presence of young stars would imply ongoing star formation in early-type galaxies, while the evolved candidates suggested that old stellar populations could be efficient UV emitters. Eventually, it became clear that the phenomenon was likely due to the presence of hot evolved stars (cf. Greggio \& Renzini 1990; Bressan, Chiosi \& Fagotto 1994; Horch, Demarque \& Pinsonneault 1992), and thus the UV upturn is still consistent with the picture of ellipticals as passively evolving populations. Observational evidence for this view was found in the spectra from the Hopkins Ultraviolet Telescope (HUT) (Ferguson et al. 1991; Brown et al. 1997); these spectra are reproduced well by a composite population of EHB, post-EHB, and post-asymptotic giant branch (post-AGB) stars. EHB stars evolve to hotter temperatures and brighter luminosities before descending the white dwarf cooling curve; their long lifetimes (compared to post-AGB stars) allows their dominance in a far-UV spectrum even when they comprise a minority of the evolved population.

Characterized by the $m_{1550}-V$ color, the UV upturn varies strongly (ranging over 2.05-4.50 mag) in nearby quiescent early-type galaxies (Bertola et al. 1982; Burstein et al. 1988), even though the spectra of ellipticals at longer wavelengths are qualitatively very similar. The $m_{1550}-V$ color is positively correlated with the strength of $\mathrm{Mg}_{2}$ line absorption (i.e. bluer at higher line strengths), opposite to the behavior of optical color indices; it also correlates with velocity dispersion and luminosity, but to a weaker extent (Burstein et al. 1988).

Because even the brightest of these galaxies are faint and extended in the UV, studies of the UV upturn have focused mostly on the composite spectral energy distributions of ellipticals. However, the UV imaging capabilities of $H S T$ have now opened the possibility of studying the resolved UV population in the nearest galaxies. Attempts to do this prior to the $H S T$ refurbishment were undertaken by King et al. (1992), for M31, and by Bertola et al. (1995), for M31 and M32. These earlier attempts suffered from significant problems with the preCOSTAR calibration. We have used the refurbished, recalibrated Faint Object Camera (FOC) to obtain deep UV images of the M31 and M32 cores. With these data we determine color-luminosity relationships for the core populations, and compare to the predictions of stellar evolutionary theories.

\section{Observations}

In February of 1994 and 1995, we obtained FOC $14 \times 14^{\prime \prime}$ UV images of the giant spiral galaxy M31 (NGC 224) and its compact elliptical companion, M32 (NGC 221). In comparison to other measured galaxies, M31 and M32 show relatively weak upturns, with respective $m_{1550}-V$ colors of 3.51 and $4.50 \mathrm{mag}$ (Burstein et al. 1988). Although the images each include the background from the bright galaxy core, we can clearly resolve many UV-bright hot stars. 
We used the STMAG system for our photometry. Extensive simulations of our FOC data show that our limiting magnitudes in the F175W and F275W bands are 24.5 and $25.5 \mathrm{mag}$, respectively. We detected 433 stars in M31 and 138 stars in M32 above our detection limits. Although our full catalog extends fainter, below these limits the completeness drops below $50 \%$ and the contamination from spurious sources becomes significant $(>10 \%)$. We show our color-magnitude diagrams in Figs. 1 and 2.

M31 has also been observed by WFPC2 with the F300W and F336W filters. 48 stars from our FOC catalog appear in these WFPC2 images, and the $F O C$-toWFPC2 colors are inconsistent with both the internal FOC and WFPC2 colors at the $0.25 \mathrm{mag}$ level. Folding archival IUE spectra of M31 and M32 through the response functions of the $F O C$ and $W F P C 2$ also shows inconsistencies at the $0.25 \mathrm{mag}$ level. However, we were unable to discern a correction that made all of these inter-instrument comparisons consistent. In any case, even if the calibration problems lie exclusively with the $F O C$, such a correction would not significantly change our results.

While the primary purpose of our observations was to study the stellar population, at the same time these new $F O C$ images provide the most detailed view of the morphology of the double nucleus of M31. WFPC observations by Lauer et al. (1993) provided the first clear optical view of the double nucleus, and deconvolved FOC UV observations (King, Stanford \& Crane 1995) indicated that the twin peaks are not due to a dust lane in the center of the galaxy. Our own images of M31 clearly show the nuclei as separate entities, also without any evidence of a dust lane. The UV-bright nucleus manifests itself as a sharp peak offset from the optically-bright nucleus. The peak is clearly not a point source, but appears to be a tight cluster of UV-bright stars. The color of the UV-bright nucleus is $m_{F 175 W}-m_{F 275 W}=-0.34 \mathrm{mag}$ within $0.2^{\prime \prime}$, consistent with stars of effective temperature $11500 \mathrm{~K}$.

\section{Results}

Our FOC observations confirm the existence of hot post-EHB stars in the centers of M31 and M32, and also confirm that post-AGB stars are not the predominant component of the UV-bright population. The existence of these EHB stars also implies that the horizontal branches in both M31 and M32 are at least somewhat extended, because "red clump" horizontal branches are unlikely to produce the stars seen in our color-magnitude diagrams.

Due to the limitations imposed by the observational uncertainties, detailed fitting of the EHB mass distribution is not warranted. Furthermore, there exist no a priori predictions of the HB mass distribution. However, we explore the simple test case of a flat distribution of mass (i.e., a constant number of stars per year per unit EHB star mass joining the zero-age $\mathrm{HB}$ ), to see if the data are consistent with a population of EHB stars. A simulation is shown in Fig. 3 . Although the simulation shows general agreement with our data, our CMDs contain a minority population $(\sim 10 \%)$ of brighter stars that cannot be explained by canonical post-EHB evolutionary tracks. Post-early-AGB or very low-mass post-AGB stars evolve through this region of the CMD, but their evolution during this phase is too rapid to produce the 35 stars seen in M31 while maintaining 


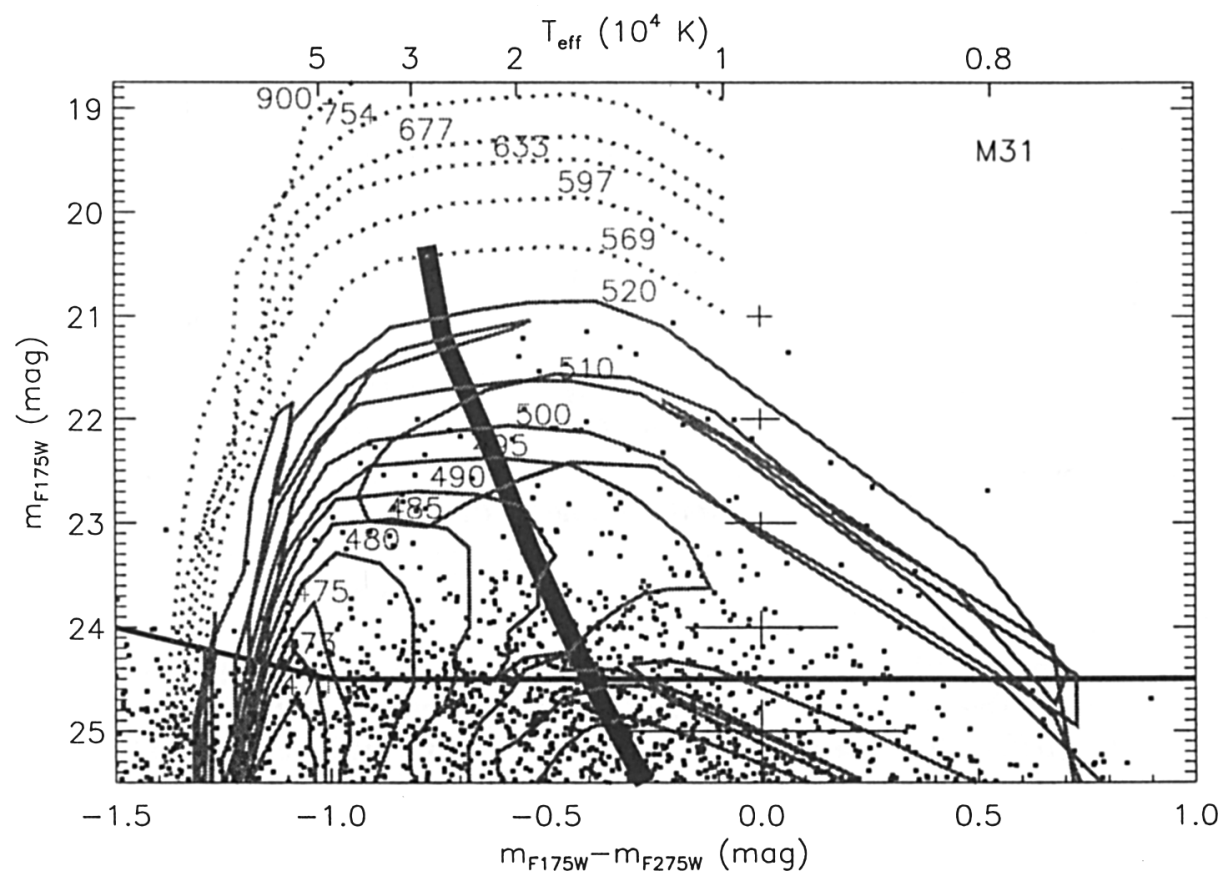

Figure 1. The color-magnitude diagram for M31 shows stars (boxes) arising from a broad distribution of masses (labels, in $\mathrm{m} M_{\odot}$ ) on the HB. The post-EHB evolutionary paths from Dorman et al. (1993) and the post-AGB paths of Vassiliadis \& Wood (1994) are shown respectively as solid and dashed light grey curves. Stars do not appear along the post-AGB tracks; for $M>0.6 M_{\odot}$, the evolution is too rapid to expect post-AGB stars above our $50 \%$ completeness limits (black horizontal curve). Stars do not cluster around the hot main sequence (dark grey vertical curve), even where the statistical uncertainty (error bars) is small. The AGB is not shown due to uncertainties in the mass loss on the AGB and in the thermally pulsing stage at the end of the AGB.

consistency with fuel consumption constraints and observed integrated spectra of M31.

The fraction of light in the resolved UV population in the center of M31 and in the center of M32 is consistent with expectations from the HUT and IUE spectra of these galaxies. The far-UV light in M31 can be explained by a main sequence population where $98 \%$ of the stars channel through intermediate-mass post-AGB evolution and $2 \%$ through EHB evolution. The far-UV light in M32 can be explained by a population where $99.5 \%$ of the stars channel through intermediate-mass post-AGB evolution and $0.5 \%$ through EHB evolution. In both galaxies, there may be an undetected contribution from very low-mass hot EHB stars, which would be below our detection limits even at their brightest luminosities. 


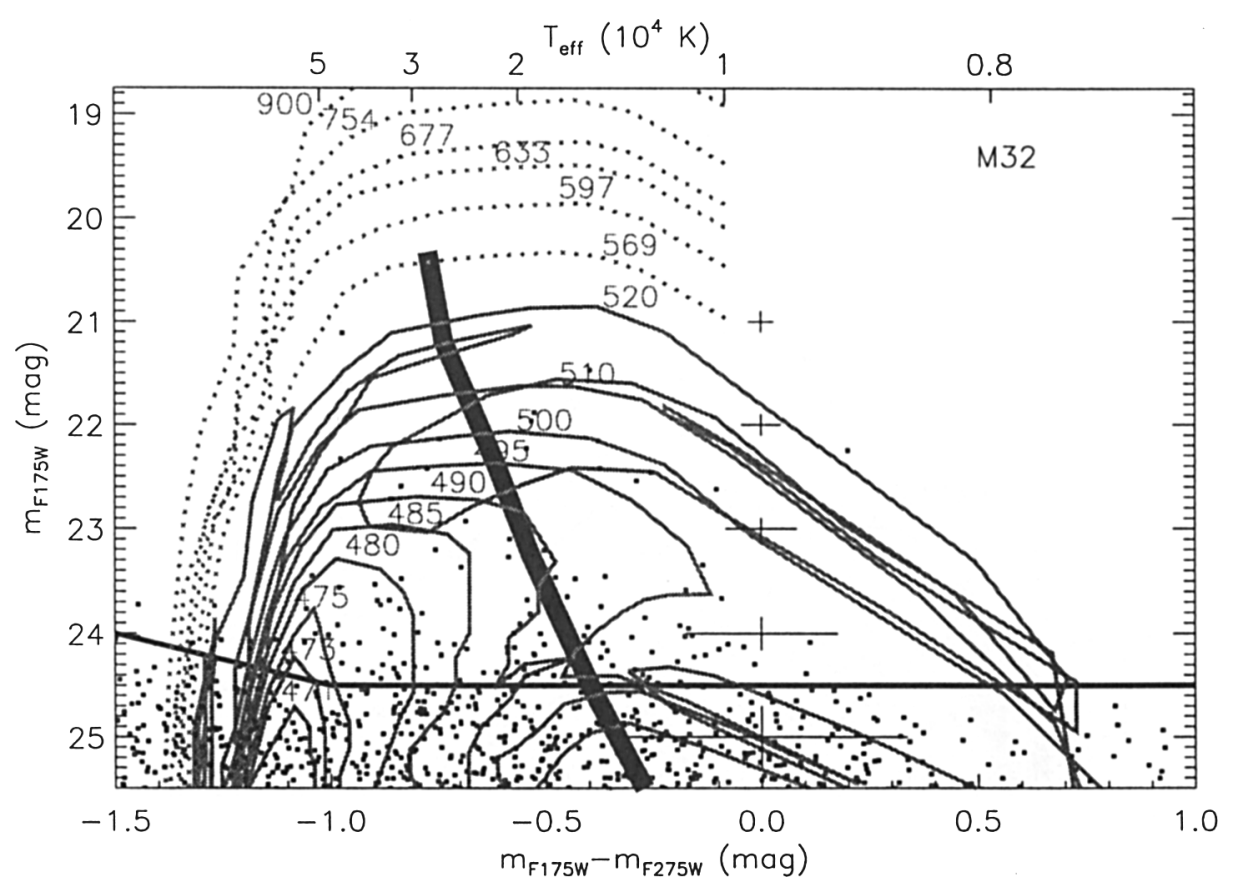

Figure 2. Relative to M31, the color magnitude diagram for M32 shows far fewer stars, but a similar distribution in temperature and luminosity.

We found that the stellar populations in M31 and M32 do not appear remarkably different in our FOC UV images. Fewer stars appear to be entering the UV-efficient EHB paths in M32, and this explains both the smaller absolute number of detected stars and the fact that we resolved a smaller fraction of the far-UV (F175W) light in M32 (6\%) as compared to M31 (18\%). The similarities of the luminosity functions and color-magnitude diagrams of M31 and M32 were unexpected, given the dramatically different $m_{1550}-V$ colors of the two galaxies. This finding suggests that, while the fractional mass in EHB stars is probably sensitive to the properties of the overall stellar population, (e.g., metallicity, age, or helium abundance), the mass distribution on the EHB may not be as sensitive to these parameters. Uncovering the full mass distribution will require deeper images that can resolve more of the evolved population. STIS observations planned for October 1998 should reach the HB in M32 and provide direct evidence for the horizontal branch distribution.

Acknowledgements. Support for this work was provided by NASA through grant GO-5435 from STScI, which is operated by AURA, Inc., under NASA contract NAS 5-26555. TMB acknowledges support at JHU by NAS 5-27000 and at GSFC by NAS 5-6499D. The work by SAS at IGPP/LLNL was performed under the auspices of the US DoE under contract W-7405-ENG-48. We thank $\mathrm{R}$. Jedrzejewski and I. King for providing insight into FOC calibration issues. 


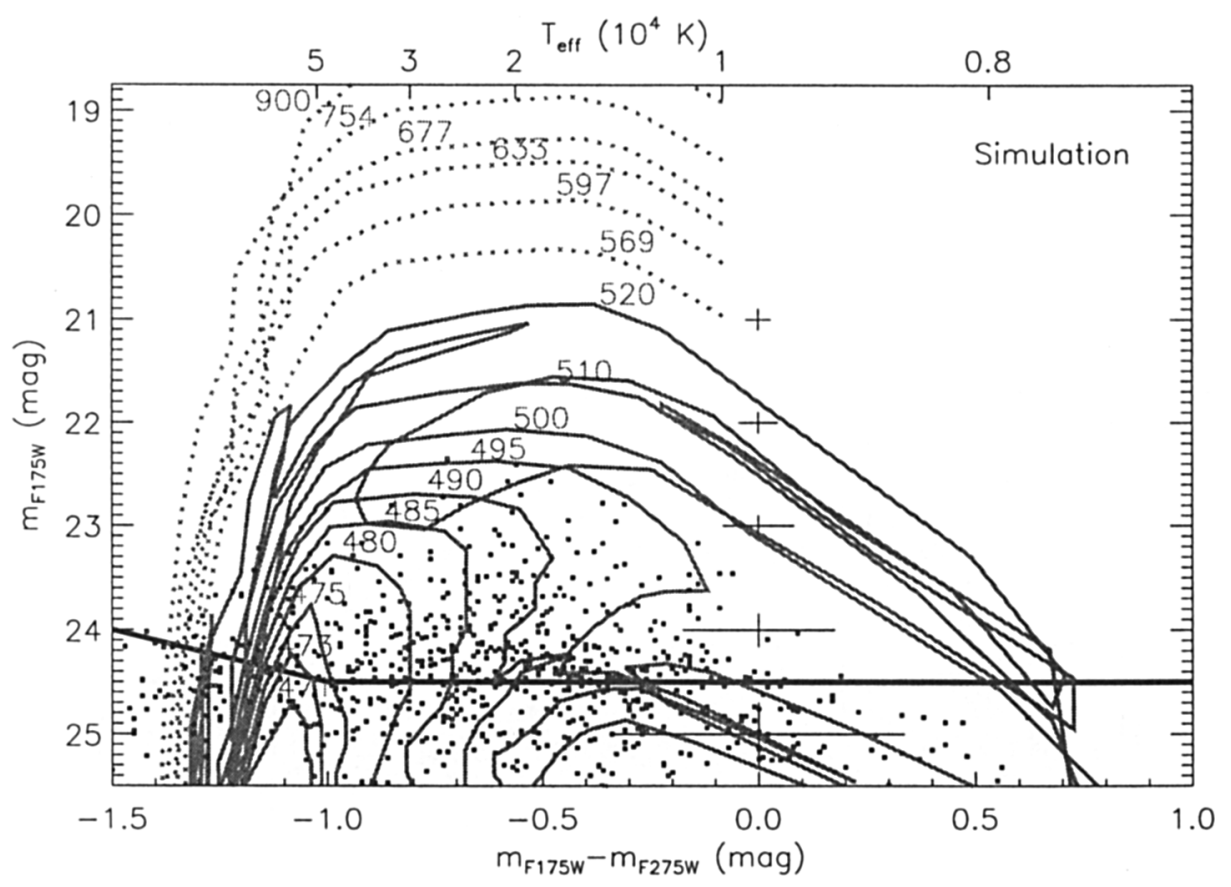

Figure 3. The "flat EHB" simulation shown here reproduces the number of stars and photometric errors in the M31 data. Although the distribution of stars is similar to that shown in Fig. 1, the brightest segments of the $0.5-0.52 M_{\odot}$ EHB tracks are not populated, because these phases are relatively short-lived.

\section{References}

Bertola, F., Capaccioli, M., Oke, J.B. 1982, ApJ, 254, 494

Bertola, F., et al. 1995, ApJ, 438, 694

Bressan, A., Chiosi, C., Fagotto, F. 1994, ApJS, 94, 63

Brown, T.M., et al. 1997, ApJ, 482, 685

Burstein, D., et al. 1988, ApJ, 328, 400

Dorman, B., Rood, R.T., O'Connell, R.W. 1993, ApJ, 419, 596

Ferguson, H.C., et al. 1991, ApJ, 382, L69

Greggio, L., Renzini, A. 1990, ApJ, 364, 35

Horch, E., Demarque, P., Pinsonneault, M. 1992, ApJ, 388, L53

King, I.R., et al. 1992, ApJ, 397, L35

King, I.R., Stanford, S.A., Crane, P. 1995, AJ, 109, 164

Lauer, T.R., et al. 1993, AJ, 106, 1436

Vassiliadis, E., Wood, P.R. 1994, ApJS, 92, 125 


\section{Discussion}

Lequeux: What is the effect of interstellar extinction on your analysis?

Brown: We assumed a foreground reddening of $E(B-V)=0.11 \mathrm{mag}$. If the foreground extinction is less (as suggested by some of the literature) or if there is also some extinction internal to the galaxies, our stars will move somewhat with respect to the tracks. But, qualitatively, our results will not change. These would still be post-EHB stars.

Pritchet: What fraction of the UV upturn flux comes from the anomalously bright post HB stars (the ones that contribute $10 \%$ of the numbers and which would not have been expected based on evolutionary time-scales)?

Brown: Only a small fraction (much less than 10\%). Most of the UV upturn comes from both the hot EHB and post-EHB. Because our detection limit is 2 mag above the ZAHB, our detected stars only account for a small fraction of the UV upturn, and these anomalous stars are only $10 \%$ of the detected population.

O'Donoghue: How did you populate the HB in your simulations? And are HBs so populated observed?

Brown: We assumed that the EHB was uniformly populated in our simulation. Although such a distribution is not observed, this simple model serves as a fiducial for comparison to our data. Given the photometric errors, and the fact that we are not resolving the HB itself, we did not feel that more complicated modelling of the HB distribution was warranted. We did investigate other distributions, but there is no way to populate the region occupied by the anomalously bright stars while matching the rest of the CMD.

Stobie: You said that the UV upturn in galaxies correlates with the metallicity in that higher metallicity galaxies have stronger upturns. If you interpret this in terms of numbers of extreme blue horizontal branch stars, what is it that produces more of these stars in more metal-rich environments?

Brown: Theorists usually assume an increase in helium abundance along with the increase in metallicity, or invoke mechanisms for increasing the mass loss on the RGB. But, no one really understands RGB mass loss and the production of EHB stars in metal-rich environments. There are only theories.

Bellazzini: Concerning your previous remark about correlating high metallicities and/or high helium content with extremely blue HB morphologies. While you can conceive that very metal rich populations generate EHB stars because of their high helium content, I think it is hazardous to postulate that solar metallicity stars have an extra mass loss such to become EHB without invoking very high helium content too. 
Brown: It is true that most high metallicity explanations for EHB stars also assume a high helium abundance (with $\Delta Y / \Delta Z>2$ ). However, there are also examples in the literature that rely upon metallicity alone (e.g., Horch, Demarque \& Pinsonneault 1992). Given the theoretical uncertainties in RGB mass loss, I do not think there is any proven explanation for the production of hot EHB stars in a metal rich population. All we know is that the UV upturn correlates with metallicity, and that the UV spectra of ellipticals and spiral bulges are well-matched by EHB synthetic spectra.

Geisler: The old open cluster, NGC 6791, with a metallicity of about +0.2 dex, does indeed contain some very faint, very blue HB stars.

Brown: True. I have mentioned NGC6791 in some papers and talks for this reason. However, there has been some talk that these blue HB stars are turning out to be binaries upon closer inspection, although I do not remember seeing such results published. 